\title{
Bioinformatic mining of kinase inhibitors that regulate autophagy through kinase signaling pathways
}

\author{
YANG YANG $^{1}$, BIAO MA ${ }^{2}$, YE JIN ${ }^{1}$, WEI BEN ${ }^{1}$, DANDAN ZHANG ${ }^{1}$, KEPING JIANG ${ }^{1}$, \\ SHUJUN FENG ${ }^{1}$, LU HUANG ${ }^{1}$ and JIANHUA ZHENG ${ }^{1}$ \\ Departments of ${ }^{1}$ Obstetrics and Gynecology and ${ }^{2}$ General Surgery, The First Affiliated Hospital \\ of Harbin Medical University, Harbin, Heilongjiang 150001, P.R. China
}

Received November 22, 2013; Accepted September 9, 2014

DOI: $10.3892 / \mathrm{mmr} .2014 .2663$

\begin{abstract}
The aim of this study was to predict the kinase inhibitors that may regulate autophagy. A total of 62 kinases were obtained through text mining by importing the keyword 'autophagy' and a 'protein kinase' Excel file to PubMed. Subsequently, 146 kinases were derivated through screening in the PubMed database by importing the 'autophagy-associated gene' and 'protein kinase' files. Following intersection of the above two methods, 54 candidate autophagy-associated kinases were obtained. Enrichment analysis indicated that these candidate autophagy-associated kinases were mainly enriched in pathways such as the calcium, Wnt, HIF-1 and mTOR signaling pathways. Among the 54 kinases, 24 were identified through text mining to have specific kinase inhibitors that regulate the corresponding functions; a total of 56 kinase inhibitors were found to be involved in the regulation of these 24 kinases. In total, nine of these 56 kinase inhibitors identified had been widely reported in autophagy regulation studies, 23 kinase inhibitors had been seldom reported and 24 had never been reported. Therefore, introducing these kinases into autophagy regulation analysis in subsequent studies may produce important results.
\end{abstract}

\section{Introduction}

Autophagy is an important process in eukaryocytes that enables the recycling of intracellular materials. During the autophagic process, certain damaged proteins or organelles are enclosed by double-membrane autophagic vacuoles and then degraded in lysosomes (animals) or in vacuoles (yeasts and plants) for reuse. Similar to apoptosis and cell aging, autophagy is a biological phenomenon of great impor-

Correspondence to: Professor Jianhua Zheng, Department of Obstetrics and Gynecology, The First Affiliated Hospital of Harbin Medical University, 23 Youzheng Street, Harbin, Heilongjiang 150001, P.R. China

E-mail: prof.jh.zheng@gmail.com

Key words: autophagy, kinase, kinase inhibitor, text mining tance, involved in numerous processes in the growth of an organism (1). Excessive or impaired autophagy may cause diseases, including cancer (2), pathogen infections (3), neurodegenerative diseases (4) and aging (5). Thus, the regulation of autophagy has become a focal point of investigation. In recent years, mTOR kinase (6), type I PI3K/Akt (7), AMPK (8), P53 (9), DAPK (10), endoplasmic reticulum annexins (11), Ire-1 (12), IP3R (13), GTPase (14), Erk1/2 (15), ceramide (16) and calcium (17), have all been observed to be involved in the regulation of autophagy. Molecular biology methods, including gene and protein analysis, have achieved marked success in studies of autophagy regulation; however, these methods are restricted in clinical practice. An approach targeting protein kinases may soon translate laboratory findings into the clinic, since an increasing number of newly developed kinase inhibitors are able to inhibit corresponding kinases with the most direct targets (18). The present study mined particular kinases regulating autophagy and predicted the kinase inhibitors that may regulate autophagy, which may provide benefits in the clinical application of autophagic regulation.

\section{Materials and methods}

Mining and screening of autophagy-associated kinases. Firstly, information regarding human protein kinases was collected from the Kinasource protein kinase database (http://kinasource.co.uk/Database/substrates.html) and an Excel file listing 511 protein kinases was produced. Using the literature-mining approach, Perl language software (www. perl.com) was used to write a literature-mining program. The retrieved keyword 'autophagy' and the 'protein kinase' Excel file were then imported to the ActivePerl 5.16.2 software (ActiveState Software Inc., Vancouver, BC, Canada) and the literature information was obtained from the National Library of Medicine's PubMed database (http://www.ncbi.nlm.nih. gov/pubmed/). The scope of the search included the titles and abstracts of all articles in the PubMed database. Valuable articles were identified and the false ones that were not related to autophagy were ruled out by manual screening.

Secondly, autophagy-associated gene ontology (GO) terms were searched for in the Gene Ontology Database (http://www. geneontology.org/), defining the species as Homo sapiens in all databases and screening out the human autophagy annota- 
tion (GO:0006914), and an 'autophagy-associated genes' Excel file was generated. Documents in PubMed were searched following the importation of the 'autophagy-associated genes' and 'protein kinase' files to the ActivePerl software, and autophagy-associated kinases concerning the corresponding autophagy-associated genes were screened out. Subsequently, the documents were checked by manual screening to verify whether the detected genes were associated with autophagy.

The kinases remaining after the intersection of the two abovementioned methods were thus proposed as candidates of the autophagy-associated kinases.

Enrichment analysis of autophagy-associated kinases. Database for Annotation, Visualization and Integrated Discovery (DAVID; http://david.abcc.ncifcrf.gov/) software was used for Kyoto Encyclopedia of Genes and Genomes (KEGG) enrichment analysis. The DAVID was opened and the kinase genes were submitted, with the whole human genome selected as the background. Subsequently, 'Functional Annotation Tool' served as the analytical tool and the results of the KEGG enrichment analysis were obtained and the pathways with $\mathrm{P} \leq 0.05$ were selected.

Regulatory network construction for autophagy-associated kinases. The corresponding signaling pathways of the specific kinases in the KEGG database (http://www.genome.jp/kegg/ pathway.html) were identified and an Excel file was set up, which contained each kinase and the corresponding KEGG signaling pathways. The file was imported to Cytoscape (version 2.6.3; http://www.cytoscape.org), with the interaction type defined as 'default interaction', the source interaction as 'kinase', and target interaction as 'KEGG signaling pathway'. The terms 'layout', 'cytoscape layout' and 'spring embedded' were then selected. A regulatory network was constructed and the kinases were connected using the same signaling pathway.

Prediction of kinase inhibitors that regulate autophagy. An Excel file of 194 kinase inhibitors was set up as determined by a database (http://www.selleckbio.com/servlet/DownloadS ervlet?fileName=Selleck-Kinase-Inhibitor-Library.xlsx). The 'autophagy-associated kinases' and 'kinase inhibitors' files were imported to the ActivePerl software and screened. The regulatory network was constructed using Cytoscape.

The texts associated with 'autophagy' and 'candidate kinase inhibitor' were extracted from the PubMed database, and the current research focus was differentiated from uninvestigated kinase inhibitors in the screened documents.

\section{Results}

Mining and screening of autophagy-associated kinases. Firstly, through text mining of 'autophagy' and 'protein kinase' terms in PubMed, 398 preliminary articles were identified and retrieved. Following manual screening, 32 articles were found to not be concerned with autophagy and were ruled out (false rate 18.09\%). In total, 62 kinases were obtained through analyzing the remaining 366 articles.

Secondly, mining for the 'Autophagy-Associated GO Term' in the Gene Ontology Database identified 56 genes with the human 'Autophagy' annotation (GO:0006914). Among these, five genes were annotated with 'Macroautophagy' (GO:0016236), 10 genes with 'Negative regulation of autophagy' (GO:0010507), seven genes with 'Negative regulation of macroautophagy' (GO:0016242), seven genes with 'Positive regulation of autophagy' (GO:0010508), 10 genes with 'Positive regulation of macroautophagy' (GO:0016239) and 16 genes with 'Regulation of autophagy' (GO:0010506). A total of 1,362 preliminary articles were retrieved in PubMed by importing the 'autophagy-associated gene' and 'protein kinase' files, and 1,026 articles remained following manual screening (false rate 24.67\%). In total, 146 kinases were obtained through analyzing these articles (Fig. 1).

Using the intersection of the two abovementioned methods, 54 candidate autophagy-associated kinases were identified (Table I).

Enrichment analysis of autophagy-associated kinases. The enrichment analysis of the 54 kinases is shown in Fig. 2, and indicates that the functions of these kinases are mainly enriched in pathways such as the calcium, Wnt, HIF-1 and mTOR signaling pathways.

Regulatory network of autophagy-associated kinases. With the further use of the KEGG database, the kinases of the same KEGG signaling pathway were connected to generate a visual network and 24 nodes signifying protein kinases of the KEGG signaling pathways were identified (See Fig. 3).

Predicted kinase inhibitors that regulate autophagy. Through text mining, 176 preliminary articles were retrieved, with 21 false articles $(11.93 \%)$ ruled out following manual screening. The remaining 155 articles indicated that, among the 54 kinases shown in Table I, 24 have specific kinase inhibitors that regulate the corresponding functions; a total of 56 kinase inhibitors were found to be involved in the regulation of these 24 kinases. A network was constructed as determined by these regulatory associations (Fig. 4).

The associations between these 'Kinase Inhibitors' and 'Autophagy' were determined through further text mining and 267 articles were screened out. A total of nine of these kinase inhibitors (wortmannin, sorafenib, geldanamycin, nocodazole, pyrimidone, rapamycin, bortezomib, cannabinoids and imatinib) had been widely reported in autophagy regulation research, 23 kinase inhibitors had been seldom reported and 24 had never been reported (Fig. 4).

\section{Discussion}

Autophagy regulates both cell survival and cell death. However, the detailed underlying mechanisms require further investigation. In the near future, preventing and controlling diseases or promoting health through the regulation of autophagy may be possible. Identifying drugs that target autophagy may be of great importance in the treatment of numerous diseases. However, whether autophagy may be accurately regulated requires further analysis.

The development of bioinformatics in the post-genomic era provides novel hypotheses in gene-to-drug research. The concept of literature-based discovery was introduced by Swanson in 1986 (19), and the study indicated that abundant undiscovered 


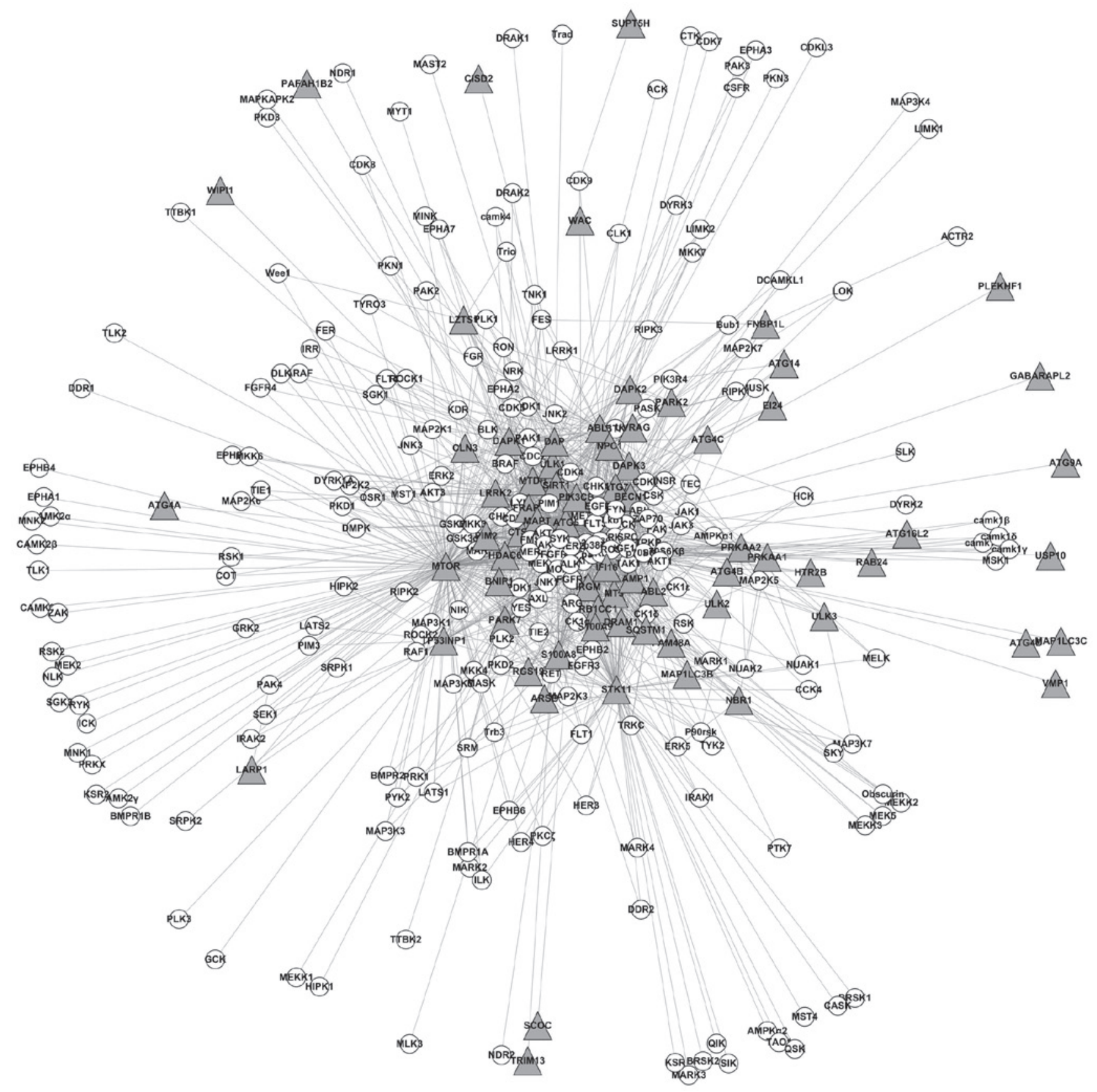

Figure 1. Network of human autophagy-related genes and autophagy-related kinases. Each human autophagy-related gene was imported into PubMed, all associated kinases were searched by text mining and the associations are presented as a network. Each triangle-shaped node refers to a gene and circular-shaped nodes indicate kinases.

public knowledge is implied in non-interactive literature sets of articles. The underlying discovery method is determined by the following principle: Certain associations between two complementary natural language text passages are largely of the form 'A causes B' and 'B causes C'. From this, it may be deduced that $\mathrm{A}$ and $\mathrm{C}$ are linked by $\mathrm{B}$, irrespective of the meaning of A or C. However, studies may not have been published concerning a possible connection between $\mathrm{A}$ and $\mathrm{C}$, although such association, if validated, may be of scientific importance. This allows for the generation of the hypotheses, such as 'Fish oil may be used for treatment of Reynaud's disease'. Currently, text mining of biomedical literature has generated a great deal of interest in biomedical research. For example, Li et al (20) detected 1,500 drug addiction-related genes using text mining; 396 closely related genes were screened through meta-analysis that identified 18 enriched signaling pathways. The authors then established an addiction-related gene database and a molecular network interactions diagram. Gajendran et al (21) identified two novel bone biology-related genes, S100A12 and FYN, through text mining and bioinformatic analysis. These two studies suggest that potential information in the literature may be identified through text mining and bioinformatic analysis. The present study, targeted at kinases, used kinase inhibitors as the intervention to determine kinase inhibitors that regulate autophagy, with the aim of more rapid translation between laboratory molecular biology results and clinical studies. 


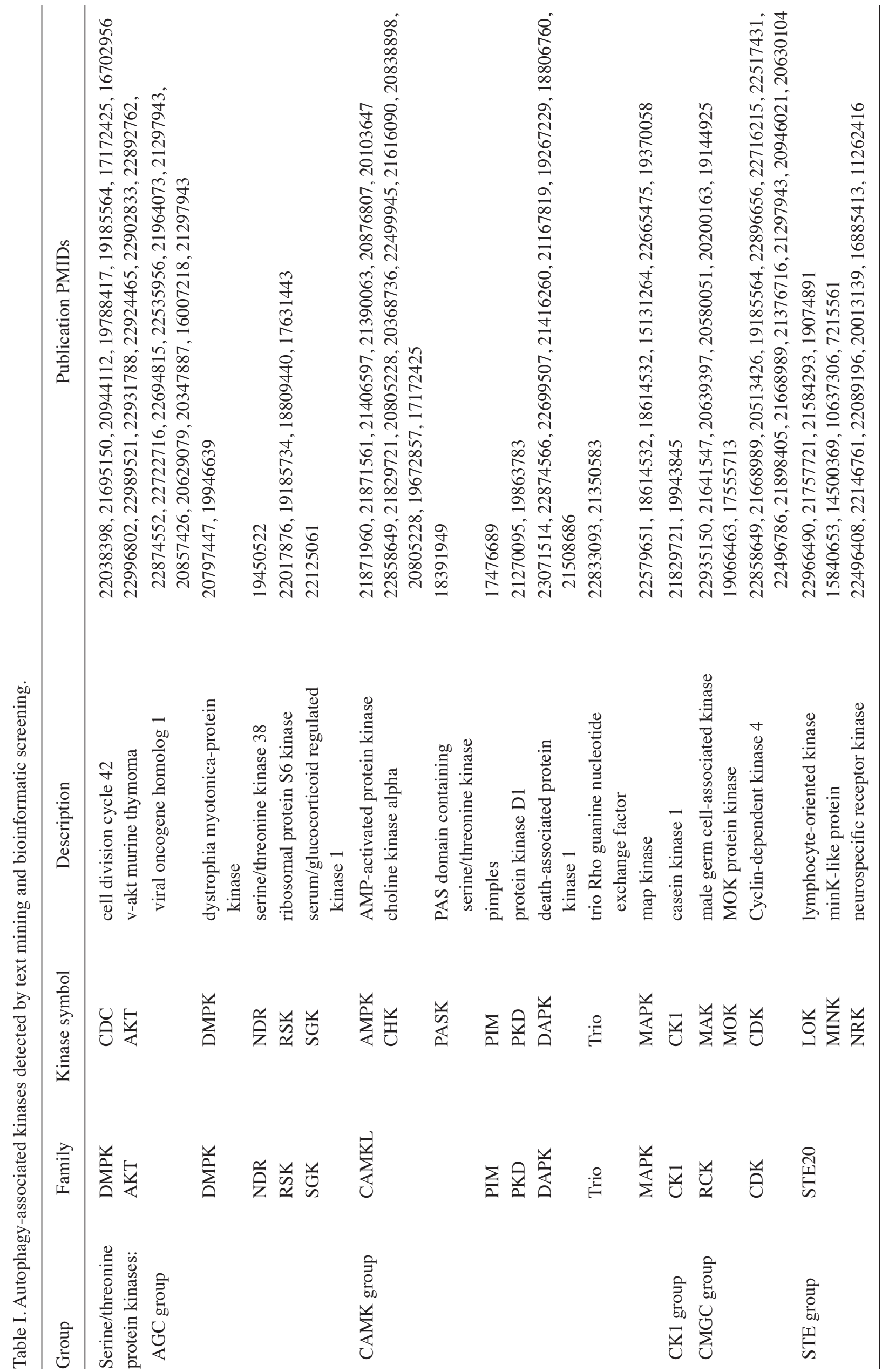




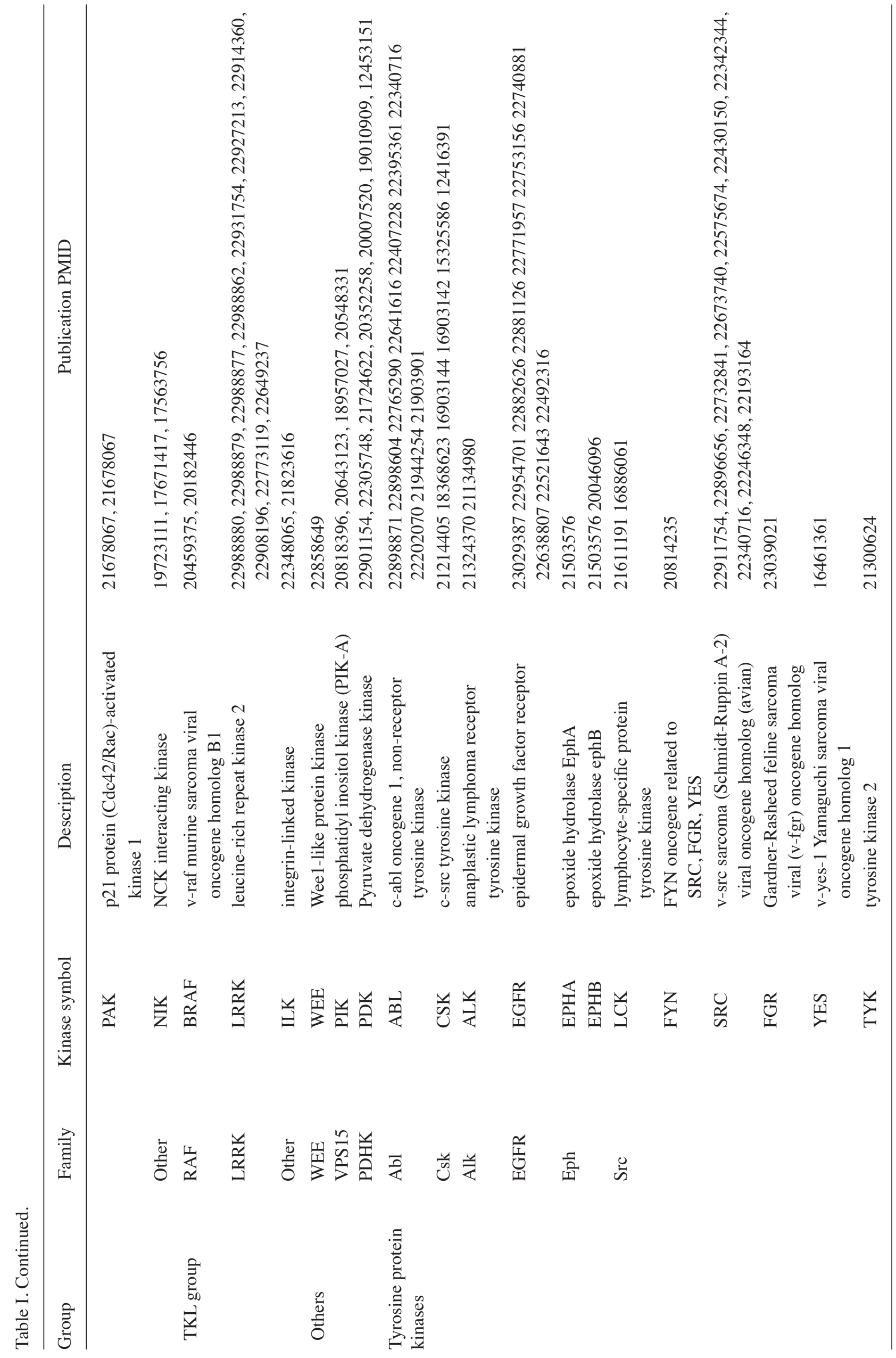




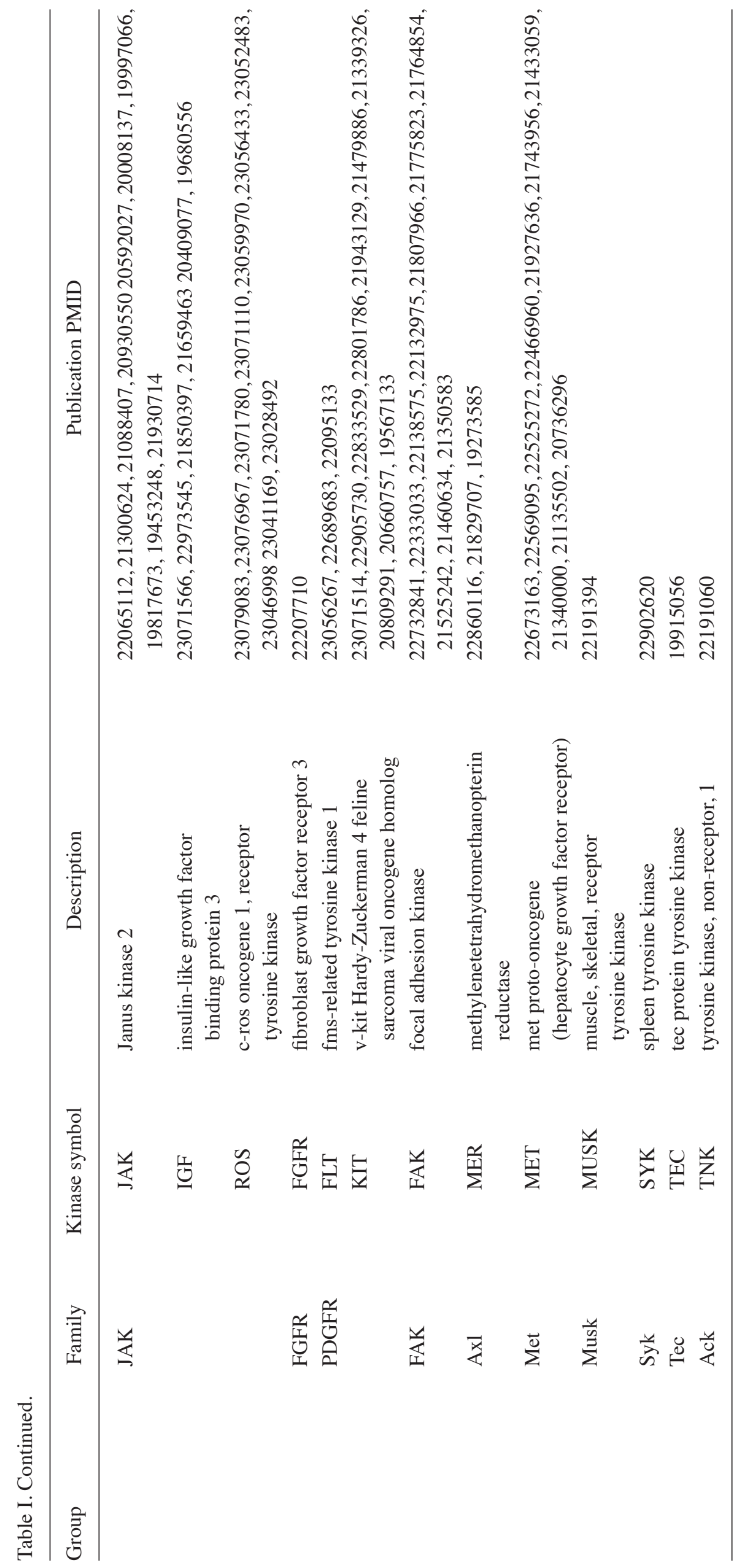




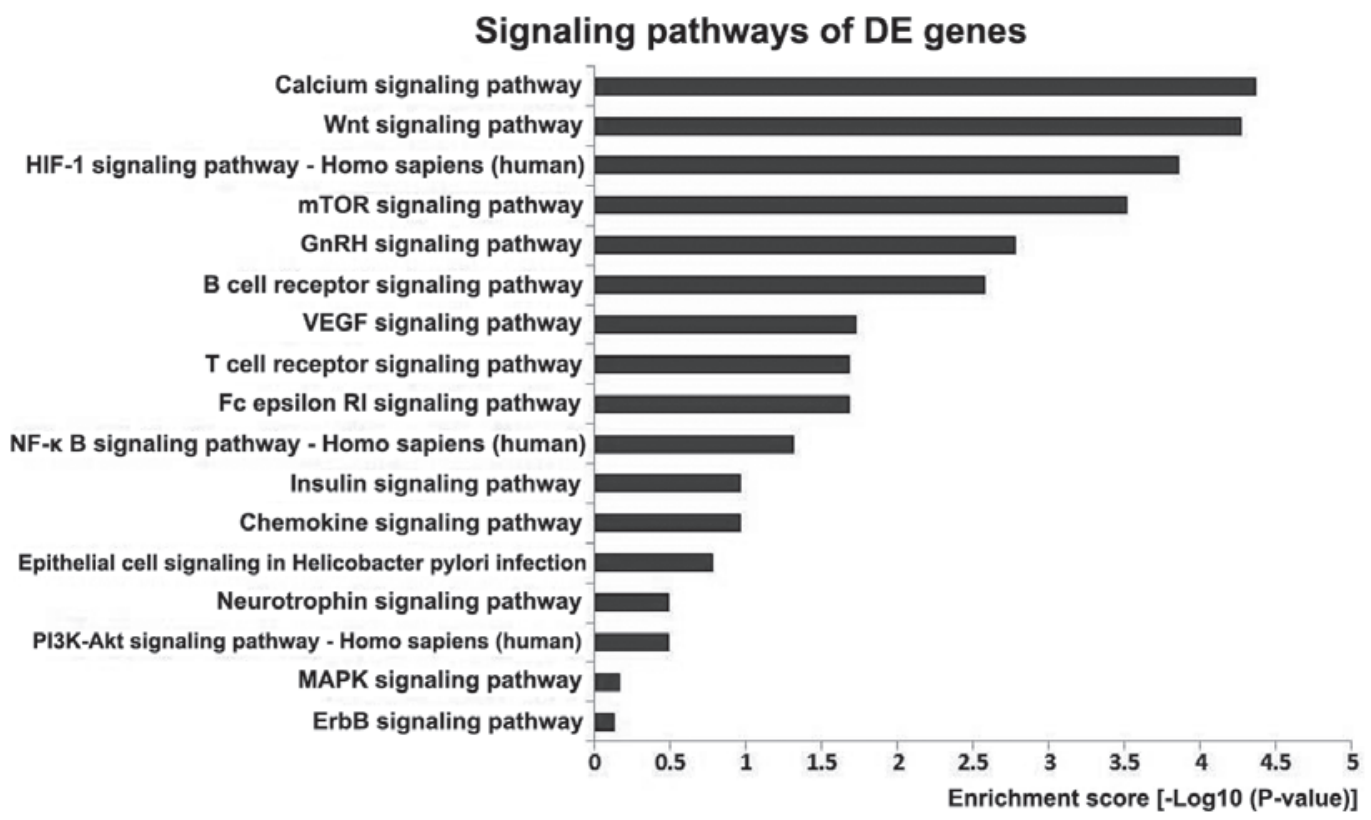

Figure 2. Signaling pathways significantly enriched by autophagy-related kinases. The $\mathrm{X}$ axis indicates the detailed significantly enriched pathway terms from the KEGG database. The Y axis is the negative log value of the enriched P-value. KEGG, Kyoto Encyclopedia of Genes and Genomes; DE, differentially expressed.

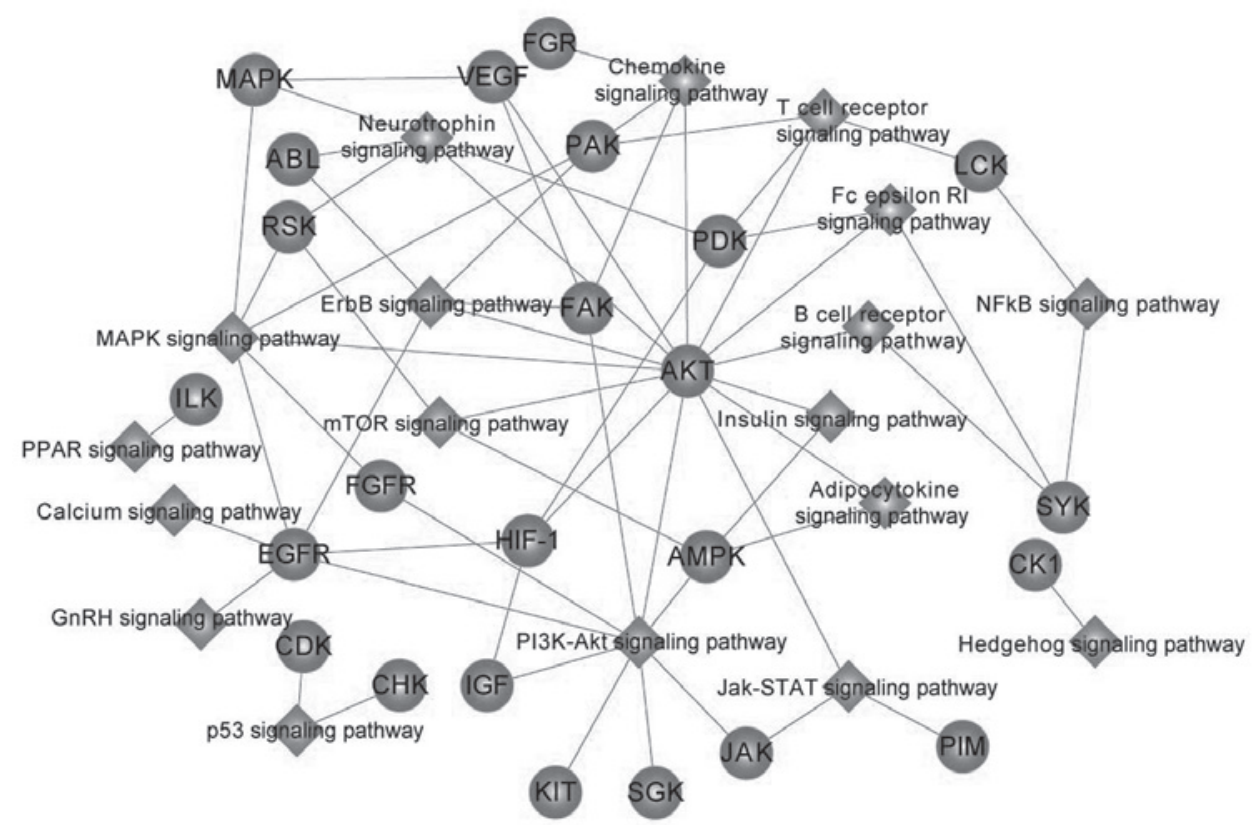

Figure 3. Functional network of autophagy-related kinases. This network is composed of autophagy-related kinases and certain related KEGG signaling pathways. The diamond-shaped nodes signify the KEGG signaling pathways and the circular-shaped nodes refer to protein kinases. KEGG, Kyoto Encyclopedia of Genes and Genomes.

In the present study, 54 autophagy-associated kinases were obtained through bioinformatic analysis, indicating that these kinases may be used as novel targets in autophagy regulation research. The KEGG signaling pathway enrichment analysis determining the functions of these kinases found that these kinases are mainly enriched in certain signaling pathways.

One study observed that calcium and calcium signaling pathways are important in autophagic regulation (22). Calcium, involved in transmembrane signal transduction, acting as the second messenger in cytoplasm, is of great importance in the regulation of cell survival and apoptosis. The autophagy induced by increases in the levels of calcium in the cytoplasm has been reported to be completed by calcium/calmodulin-dependent protein kinase kinase $\beta$-mediated AMPK activation and mTOR inhibition (23). Grotemeier et al (24) revealed that calcium induces autophagy independently, without calcium-mediated AMPK activation or complete mTOR inhibition. The fact that the increase 


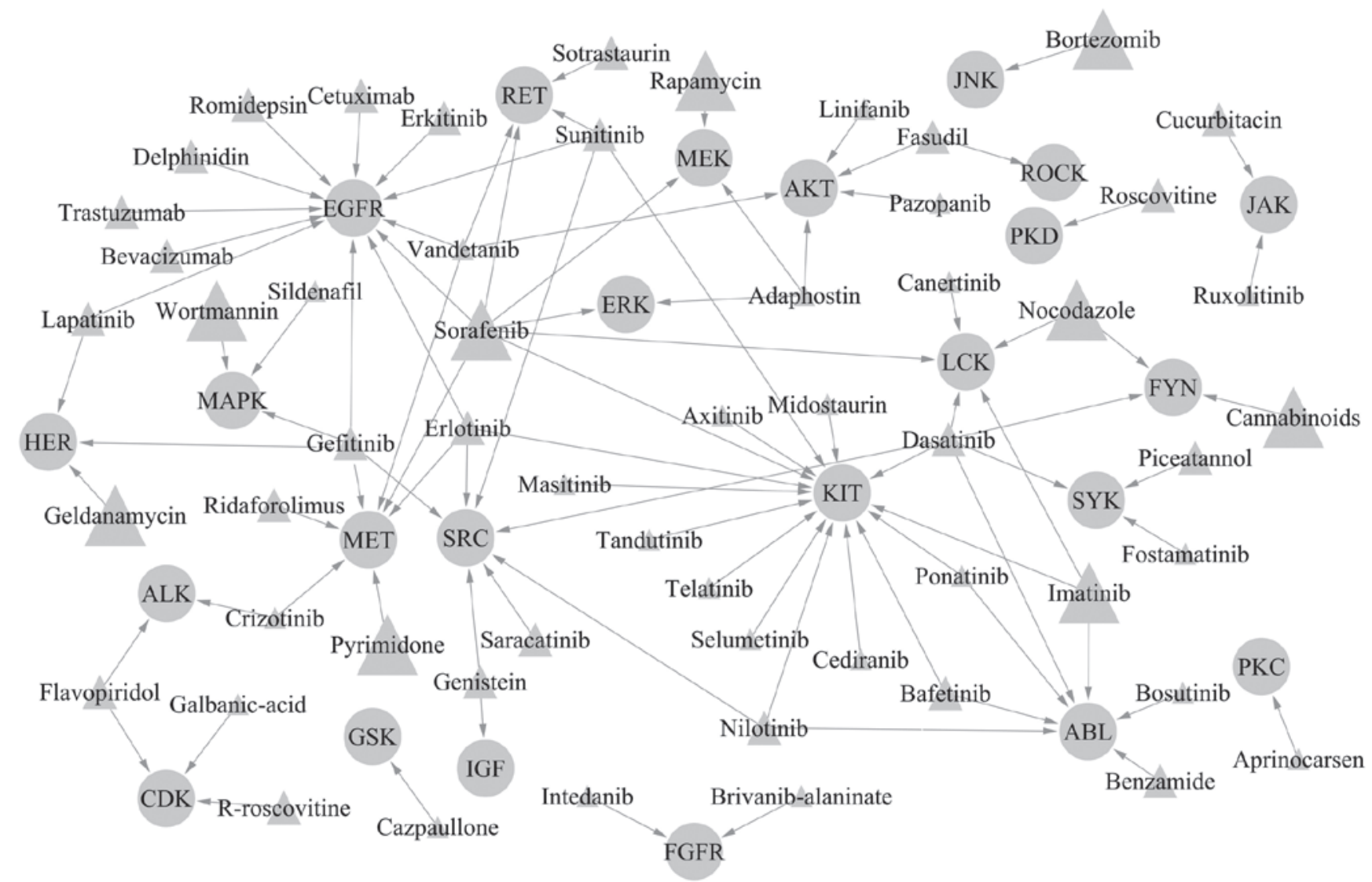

Figure 4. Network of human autophagy-related kinases and corresponding kinase inhibitors. Each circular-shaped node refers to a kinase; each triangle-shaped node indicates a kinase inhibitor. The arrows indicate regulation. Large triangles indicate $>11$ studies in PubMed regarding regulation of autophagy by the kinase inhibitor, medium-sized triangles signify 1-10 studies and small triangles represent no studies.

in the levels of calcium in the cytoplasm induce autophagy and hinder the degradation of autophagic vacuoles indicates that calcium induces autophagy independently without the aid of other medications. DAPK is a calcium/calmodulin serine/threonine kinase, acting as a tumor suppressor gene, promoting cell apoptosis and autophagy. Beclin-1 is a protein with a BH3 domain. In general, Beclin-1 combines with the BH3 domain of the apoptosis protein bcl-2/bcl-XL inhibitor and DAPK phosphorylates the BH3 Thr119 site in Beclin-1, resulting in the dissociation of Beclin-1 and Bcl-XL, inducing autophagy (25). All studies verified that the calcium signaling pathway regulates autophagy, which is in accordance with the present study.

Protein kinases, also termed protein phosphokinases, are associated with almost all cell functions. However, in particular, kinases are important in signaling between cells and the execution of complicated cell functions, including cell division. Thus, kinase over-activity may result in the development of certain types of cancer (26). The key role of kinases in controlling cell behavior has been widely investigated as a target in the treatment of numerous other diseases, including diabetes (27), osteoporosis (28), inflammation (29) and ophthalmopathy (30).

Techniques such as gene transfection or RNA intervention have been shown to be distinctive and effective in the laboratory setting for regulating kinases as targets of autophagy research, but these methods have a number of problems when translated into clinical practice. However, various emerging kinase inhibitors available on the market have been observed to be effective in regulating kinase activity (31). Thus, introducing kinase inhibitors into switching autophagy may have more practical applications, and this may speed up the translation between laboratory results and clinical application, in compliance with the concept of translational medicine.

Kinase inhibitors, compounds that inhibit protein kinase activity, have been commonly analyzed in recent new drug studies and an increasing number of drugs have emerged. In recent years, a number of large pharmaceutical companies have developed novel drugs with the primary goal of targeting kinases (32). In total more than 30 kinase inhibitors have undergone clinical studies and been approved by the Food and Drug Administration of the USA. First generation products, such as imatinib (33), gefitinib (34) and erlotinib (35), have been shown to be effective in inhibiting the activity of the targeted kinases. Second generation AbL kinase-targeted drugs, including dasatinib, have been subsequently developed (36). Third generation Aurora kinase inhibitors remain under investigation (37). This describes only a fraction of current kinomic studies, as a great number of novel compounds are being developed.

The present study identified nine kinase inhibitors (wortmannin, sorafenib, geldanamycin, nocodazole, pyrimidone, rapamycin, bortezomib, cannabinoids and imatinib) that have 
been widely reported in autophagy regulation research. Kinase inhibitors have previously been shown to regulate autophagy.

However, other kinase inhibitors that regulate autophagy were also identified in the present study. Among the 56 kinase inhibitors derived from the bioinformatic analysis, 24 (crizotinib, galbanic-acid, cazpaullone, intedanib, brivanib-alaninate, sotrastaurin, linifanib, midostaurin, ponatinib, fostamatinib, cediranib, bosutinib, aprinocarsen, pazopanib, adaphostin, canertinib, ruxolitinib, axitinib, telatinib, selumetinib, vandetanib, tandutinib, sildenafil and masitinib) were not found to have been previously reported with regard to autophagy regulation in PubMed. This indicates that introducing these kinases into autophagy regulation analysis in later studies may produce important benefits in the future.

\section{References}

1. Zou Y, Wang Q, Li B, Xie B and Wang W: Temozolomide induces autophagy via ATM-AMPK-ULK1 pathways in glioma. Mol Med Rep 10: 411-416, 2014.

2. Wang W, Fan H, Zhou Y, et al: Knockdown of autophagy-related gene BECLIN1 promotes cell growth and inhibits apoptosis in the A549 human lung cancer cell line. Mol Med Rep 7: $1501-1505,2013$

3. Richards AL and Jackson WT: Intracellular vesicle acidification promotes maturation of infectious poliovirus particles. PLoS Pathog 8: e1003046, 2012.

4. Kingwell K: Genetics: Mutations in autophagy gene cause a rare and severe neurodegenerative disease. Nat Rev Neurol 9: 182, 2013

5. Salminen A, Ojala J, Kaarniranta K and Kauppinen A: Mitochondrial dysfunction and oxidative stress activate inflammasomes: impact on the aging process and age-related diseases. Cell Mol Life Sci 69: 2999-3013, 2012.

6. Zou Z, Yuan Z,Zhang Q,et al: Aurora kinase A inhibition-induced autophagy triggers drug resistance in breast cancer cells. Autophagy 8: 1798-1810, 2012.

7. Chen A, Xiong LJ, Tong Y and Mao M: Neuroprotective effect of brain-derived neurotrophic factor mediated by autophagy through the PI3K/Akt/mTOR pathway. Mol Med Rep 8: 1011-1016, 2013

8. Mack HI, Zheng B, Asara JM and Thomas SM: AMPK-dependent phosphorylation of ULK1 regulates ATG9 localization. Autophagy 8: 1197-1214, 2012.

9. Zhao YG, Zhao H, Miao L, et al: The p53-induced gene Ei24 is an essential component of the basal autophagy pathway. J Biol Chem 287: 42053-42063, 2012

10. Gandesiri M, Chakilam S, Ivanovska J, et al: DAPK plays an important role in panobinostat-induced autophagy and commits cells to apoptosis under autophagy deficient conditions. Apoptosis 17: 1300-1315, 2012

11. Yadunandam AK, Yoon JS, Seong YA, Oh CW and Kim GD: Prospective impact of 5-FU in the induction of endoplasmic reticulum stress, modulation of GRP78 expression and autophagy in Sk-Hep1 cells. Int J Oncol 41: 1036-1042, 2012.

12. Roy A and Kolattukudy PE: Monocyte chemotactic protein-induced protein (MCPIP) promotes inflammatory angiogenesis via sequential induction of oxidative stress, endoplasmic reticulum stress and autophagy. Cell Signal 24: 2123-2131, 2012.

13. Criollo A, Maiuri MC, Tasdemir E, et al: Regulation of autophagy by the inositol trisphosphate receptor. Cell Death Differ 14: 1029-1039, 2007.

14. Lipatova Z, Belogortseva N, Zhang XQ, et al: Regulation of selective autophagy onset by a Ypt/Rab GTPase module. Proc Natl Acad Sci USA 109: 6981-6986, 2012.

15. Cea M, Cagnetta A, Fulciniti M, et al: Targeting NAD ${ }^{+}$salvage pathway induces autophagy in multiple myeloma cells via mTORC1 and extracellular signal-regulated kinase (ERK1/2) inhibition. Blood 120: 3519-3529, 2012.
16. Meijer AJ and Codogno P: Signalling and autophagy regulation in health, aging and disease. Mol Aspects Med 27: 411-425, 2006.

17. Crawford SE, Hyser JM, Utama B and Estes MK: Autophagy hijacked through viroporin-activated calcium/calmodulindependent kinase kinase- $\beta$ signaling is required for rotavirus replication. Proc Natl Acad Sci USA 109: E3405-E3413, 2012.

18. Zhang K, Wang X and Wang H: Effect and mechanism of Src tyrosine kinase inhibitor sunitinib on the drug-resistance reversal of human A549/DDP cisplatin-resistant lung cancer cell line. Mol Med Rep 10: 2065-2072, 2014.

19. Swanson DR: Fish oil, Raynaud's syndrome, and undiscovered public knowledge. Perspect Biol Med 30: 7-18, 1986.

20. Li CY, Mao X and Wei L: Genes and (common) pathways underlying drug addiction. PLoS Comput Biol 4: e2, 2008.

21. Gajendran VK, Lin JR and Fyhire DP: An application of bioinformatics and text mining to the discovery of novel genes related to bone biology. Bone 40: 1378-1388, 2007.

22. Cárdenas $\mathrm{C}$ and Foskett JK: Mitochondrial $\mathrm{Ca}(2+)$ signals in autophagy. Cell Calcium 52: 44-51, 2012.

23. Høyer-Hansen M, Bastholm L, Szyniarowski P, et al: Control of macroautophagy by calcium, calmodulin-dependent kinase kinase-beta, and Bcl-2. Mol Cell 25: 193-205, 2007.

24. Grotemeier A, Alers S, Pfisterer SG, et al: AMPK-independent induction of autophagy by cytosolic $\mathrm{Ca}^{2+}$ increase. Cell Signal 22: 914-925, 2010.

25. Zalckvar E, Berissi H, Mizrachy L, et al: DAP-kinase-mediated phosphorylation on the $\mathrm{BH} 3$ domain of beclin 1 promotes dissociation of beclin 1 from Bcl-XL and induction of autophagy. EMBO Rep 10: 285-292, 2009.

26. Lu W, Qu JJ, Li BL, et al: Overexpression of p21-activated kinase 1 promotes endometrial cancer progression. Oncol Rep 29: 1547-1555, 2013.

27. Sajan MP, Nimal S, Mastorides S, et al: Correction of metabolic abnormalities in a rodent model of obesity, metabolic syndrome, and type 2 diabetes mellitus by inhibitors of hepatic protein kinase C-1. Metabolism 61: 459-469, 2012.

28. Liu Y, Berendsen AD, Jia S, et al: Intracellular VEGF regulates the balance between osteoblast and adipocyte differentiation. J Clin Invest 122: 3101-3113, 2012.

29. Kim SB, Kang OH, Joung DK, et al: Anti-inflammatory effects of tectroside on UVB-induced HaCaT cells. Int J Mol Med 31: 1471-1476, 2013.

30. Murakami Y, Matsumoto H, Roh M, et al: Receptor interacting protein kinase mediates necrotic cone but not rod cell death in a mouse model of inherited degeneration. Proc Natl Acad Sci USA 109: 14598-14603, 2012.

31. Marech I, Patruno R, Zizzo N, et al:Masitinib (AB1010), from canine tumor model to human clinical development: where we are? Crit Rev Oncol Hematol 91: 98-111, 2014.

32. Ferrè F, Palmeri A and Helmer-Citterich M.Computational methods for analysis and inference of kinase/inhibitor relationships.Front Genet 30: 196, 2014.

33. Niu CC, Zhao C, Yang ZD, et al: Downregulation of $\gamma$-catenin inhibits CML cell growth and potentiates the response of CML cells to imatinib through $\beta$-catenin inhibition. Int J Mol Med 31: 453-458, 2013.

34. Wu J, Min R, Wu M and Chen W: Gefitinib induces mitochondrialdependent apoptosis in Saccharomyces cerevisiae. Mol Med Rep 4: 357-362, 2011.

35. Guttman-Yassky E, Mita A, De Jonge M, et al: Characterisation of the cutaneous pathology in non-small cell lung cancer (NSCLC) patients treated with the EGFR tyrosine kinase inhibitor erlotinib. Eur J Cancer 46: 2010-2019, 2010.

36. Song Z, Lu P, Furman RR, et al: Activities of SYK and PLCgamma2 predict apoptotic response of CLL cells to SRC tyrosine kinase inhibitor dasatinib. Clin Cancer Res 16: 587-599, 2010.

37. Li Y, Tang K, Zhang H, et al: Function of Aurora kinase A in Taxol-resistant breast cancer and its correlation with P-gp. Mol Med Rep 4: 739-746, 2011. 